\title{
A multilevel approach to botulinum toxin type $A$ treatment of the (ilio)psoas in spasticity in cerebral palsy
}

\author{
Guy Molenaers ${ }^{a}$, Marijke Eyssen ${ }^{\mathrm{b}}$, Kaat Desloovere ${ }^{\mathrm{c}}$, llse Jonkers ${ }^{\mathrm{d}}$ \\ and Paul De Cock ${ }^{\mathrm{C}}$ \\ ${ }^{a}$ Department of Orthopaedic Surgery, 'D Department of Neuro Paediatrics, \\ 'Gait Analysis Laboratory, 'Department of Physical Therapy, Faculty of Physical \\ Education and Physical Therapy, University Hospital Leuven, Belgium
}

Correspondence to Dr Guy Molenaers, Department of Orthopaedic Surgery, University Hospital Leuven, UZ Pellenberg, Weligerveld 1, 3212 Pellenberg, Belgium

Tel: +32 1633 8800; fax: +32 16338824

\begin{abstract}
In spasticity, flexion deformity of the hip is frequently associated with contracture or hyper-reflexia of the psoas muscle. Botulinum toxin type A (BTX-A) has been used for some considerable time in the management of paediatric gait disorders. We have been using a multilevel approach to manage spasticity in cerebral palsy for several years, the combination of gait analysis and clinical evaluation being important for the selection of target muscles for BTX-A injections. Twenty cerebral palsy children (12 female) with spasticity were treated with BTX-A injections (BOTOX ${ }^{\varpi}$;ean dose, 2 U/kg body weight) into the psoas muscle. Patients were monitored using range of motion measurements of maximal hip extension, clinical estimates of hypertonia in the hip flexors, gait analysis (three-dimensional kinematics and kinetics) and surface electromyography of major lower limb muscles. Full gait analysis was carried out on 12 of the patients. Significant clinical improvements were observed following 15 of the 21 psoas treatments. Furthermore, the kinematics results of gait analysis showed improvement in one or more parameters in nine of the 12 patients. In conclusion, we have demonstrated the value of a multilevel approach to BTX-A treatment in the management of spasticity in children with cerebral palsy. Eur J Neurol 6 (suppl 4):S59-S62 0 Lippincott Williams \& Wilkins
\end{abstract}

Keywords: cerebral palsy, spasticity, children, botulinum toxin type A, psoas, multilevel

\section{INTRODUCTION}

In patients with spastic involvement, flexion deformity of the hip is frequently associated with contracture or hyper-reflexia of the psoas muscle. A number of treatment options have been used in an attempt to facilitate hip extension during the terminal stance phase of gait and/or to correct the position of the pelvis in the sagittal plane, the treatment selection depending upon the exact nature of the condition.

The use of botulinum toxin type A (BTX-A) in the management of paediatric gait disorders has now gained widespread acceptance. At the University Hospital Pellenberg, Leuven, we have been following a multilevel approach to the management of spasticity with cerebral palsy for several years (Jonkers et al., 1997). Both gait analysis and clinical evaluation are considered to be critical factors in the selection of target muscles for injection. This multilevel approach highlighted the issue of including the psoas as a target muscle in the injection sequence. Furthermore, the role of BTX-A injections in the psoas muscle in combination with multilevel surgery was explored.

In this paper, we describe the BTX-A injection technique that we are currently using for the psoas muscle. In addition, functional outcome results are presented, which demonstrate the positive effect of BTX-A injections into the psoas muscle as part of our multilevel treatment strategy.

\section{METHODS}

Study population

Over an 18-month period, 20 cerebral palsy children with spasticity (12 female, eight male) were recruited for BTX-A treatment: six patients were unable to walk, 
seven could walk using aids, and seven patients were able to walk unaided. All patients were aged between 3 and 9 years, except for one 16-year-old. Ten patients had quadriplegia, five had severe diplegia (high tone, poor selectivity and power), three had mild diplegia and two patients had hemiplegia.

\section{Clinical assessment}

All the patients treated with BTX-A injections were evaluated using an extensive clinical examination, which included the range of motion of maximal hip extension (measured in degrees), and a clinical estimation of the level of hypertonia in the hip flexors $(0$, no hypertonia; 1 , mild hypertonia; 2 , moderate hypertonia; 3 , severe hypertonia). In addition, gait analysis was performed: three-dimensional kinematics and kinetics (using a six-camera Vicon datacapturing system and two Advanced Mechanical Technology, Inc., force-plates) and surface electromyography of seven major lower limb muscles. An increase in range of motion of $\geq 5^{\circ}$ for the clinical measurement of hip extension and/or an improvement of one on the hypertonia score of the hip flexors postinjection was judged to be a clinically significant improvement.

Full gait analysis was performed on 12 patients. For children younger than 4 years of age, or for children unable to walk unaided, the gait analysis involved a normal video recording with a split screen (four-camera) and a freeze-frame facility. This level of analysis was used for eight children (one child twice). Gait analysis was used before and 2 months after BTX-A injections.

Under general anaesthesia, prior to BTX-A injection, an additional clinical examination was performed to differentiate the static and dynamic components of muscle hypertonia.

\section{Indications}

Based on the clinical goals used to determine the target muscles for multilevel surgery, clinical goals for the use of BTX-A injections in the psoas were:

- an increased angular velocity of hip flexion at preswing and initial swing (as a response to the stretch of the hip flexors at the end of terminal stance);

- an increased peak hip flexion during swing;

- an increased pelvic anteversion during the gait cycle;

- a decreased hip extension at terminal stance; and

- the presence of a dynamic hip flexion contracture, as identified in the clinical examination.

For kinematics of gait analysis in the sagittal plane $(n=12)$, the following items were evaluated:
- changes in angular velocity of hip flexion;

- changes in pelvic anteversion;

- changes in peak hip flexion at swing phase; and

- changes in maximal hip extension.

\section{TREATMENT PROCEDURE AND DOSAGE}

BTX-A (BOTOX ${ }^{\circledR}$, Allergan Inc., Irvine, CA, USA) was prepared using the standard procedure and injected at multiple sites in the psoas muscle. The mean dose of BTX-A for the psoas muscle was $2 \mathrm{U} / \mathrm{kg}$ body weight (range, 1-4 U/kg body weight).

The anterior superior iliac spine and iliac crest were identified for BTX-A injections into the psoas. The psoas muscle was palpated by moving the finger from lateral to medial, pressing firmly: the muscle was identified as a bump. As with other injected muscles, the location of the needle was verified by moving the limb (flexing and extending the hip), causing the needle to move simultaneously with the muscle. The initial BTX-A injections into the psoas were performed using ultrasonography to confirm correct injection placement. (Concomitant injection of the iliacus muscle could not always be avoided.) Injections into the psoas were performed bilaterally in 12 patients and unilaterally in eight patients. The medial hamstrings were injected in all patients. In 17 cases, the adductor longus was also included and the gastrocnemius was injected in 15 cases.

All patients followed a post-treatment physiotherapy programme which consisted of three to five sessions per week.

\section{RESULTS}

In total, 21 treatment sessions involving the psoas were performed $(n=20$; one patient was injected twice). A significant clinical improvement was observed in 15 of the 21 treatment sessions, as assessed using hip extension measurements and hypertonia scores of the hip flexors. No change was reported for five cases and no information was available for one case. For the nine patients for whom full gait analysis was not available, hip extension was judged to be improved in six children using video analysis, as assessed by two independent observers.

The kinematic gait analysis results of the 12 patients measured showed improvement in one or more of the parameters for the sagittal plane in nine of them, and no improvement in the remaining three patients (Figures 1 and 2). Figure 3 shows the improvements in hip extension following injection of the psoas only, and allows comparison with multilevel injections that include the psoas. 


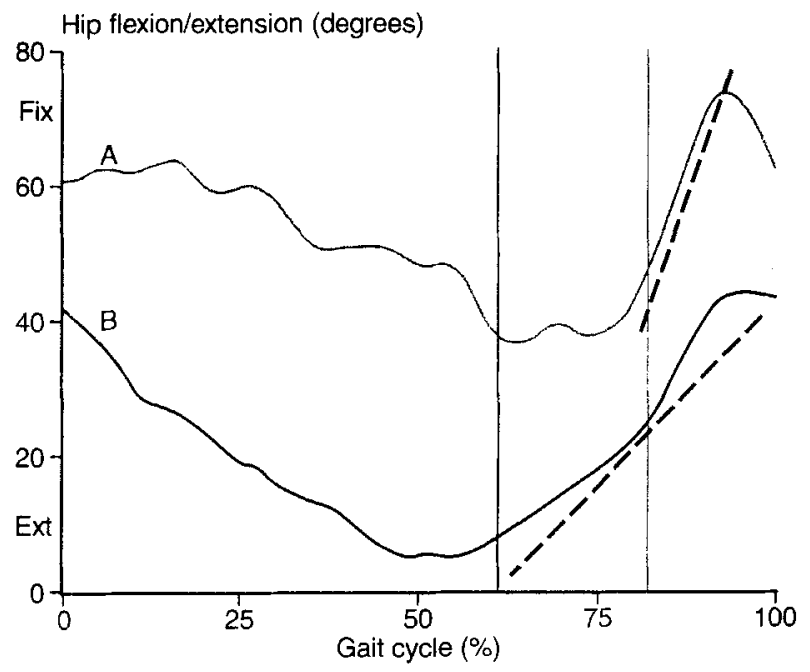

FIGURE 1. Gait analysis kinematics of the hip joint of a 6-year-old boy, sagittal plane, (A) before and (B) after multilevel botulinum toxin type $A$ injections. The gradients of the straight hashed lines were used to calculate the velocity of hip flexion during initial swing

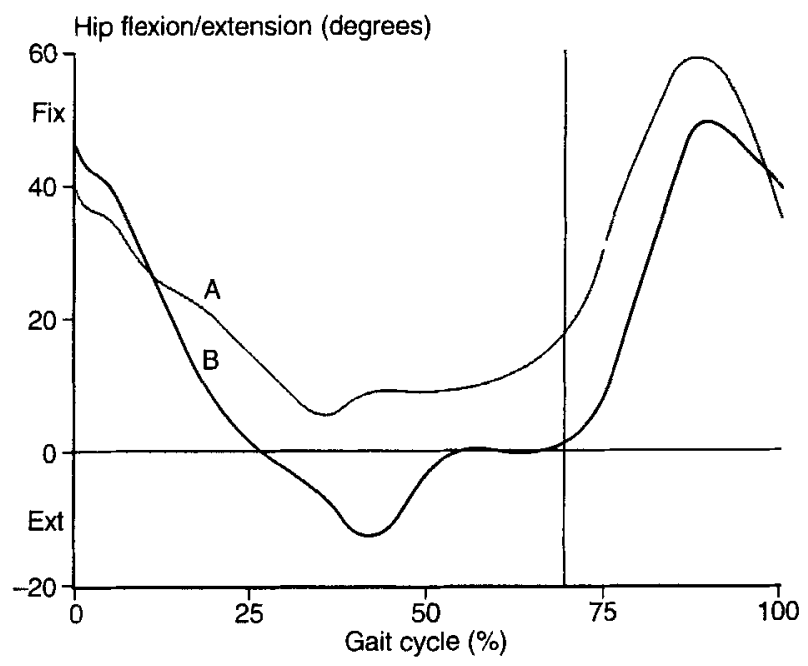

FIGURE 2. Gait analysis kinematics of the hip joint of a 7-year-old girl, sagittal plane, (A) before and (B) after multilevel botulinum toxin type $A$ injections

\section{DISCUSSION}

We have shown that a multilevel approach to treatment with BTX-A is very useful in the management of spasticity in children with cerebral palsy. Clinical evaluation and gait analysis enables evaluation of the necessity for BTX-A treatment of the psoas and assessment of the functional effect of BTX-A injec-

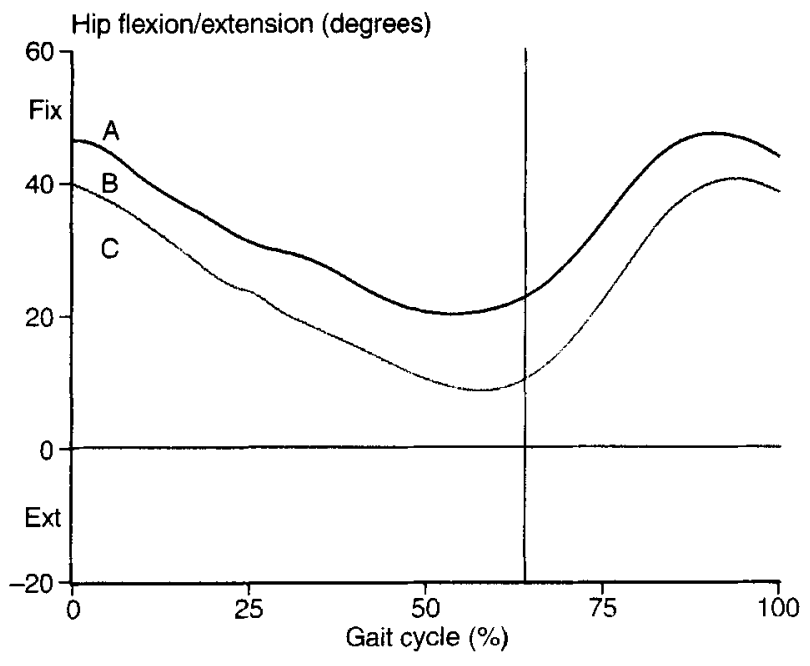

FIGURE 3. Gait analysis kinematics of the hip joint of a 20-year-old girl, not included in the study, who underwent a single-level botulinum toxin type $A(B T X-A)$ injection in the psoas; kinematics in the sagittal plane, $(A)$ before and (B) after single-level BTX-A injection. (C) A normal gait cycle for comparison

tions. Although all the children in the study were subjected to the multilevel treatment, which consequently addressed the medial hamstrings, we believe that the observed kinematic changes in hip position can be at least partially attributed to the injection of the (ilio)psoas. In support of this finding, improvements in hip extension were observed following isolated (ilio)psoas injections.

We recognise that for some children, no improvement was achieved. However, these were children with severe spasticity in whom slight changes in spasticity might not easily have been observed. In addition, the clinical re-evaluation interval of 2 months may not have been long enough to detect changes in range of motion at the hip. These factors may also explain the results of the kinematic evaluation for these patients.

Based on these preliminary results, we conclude that BTX-A injections into the psoas appear to be effective in resolving the major functional deficits caused by psoas hypertonicity. We believe that inclusion of injection of the psoas muscle within a multilevel BTX-A treatment schedule can be considered a safe treatment option to avoid fixed contractures. Furthermore, a combination of multilevel surgery and BTX-A injections into the psoas might be an optimal solution for cases in which psoas release or lengthening are contraindicated. 


\section{REFERENCE}

Jonkers I, Molenaers G, Eyssen M, Greive A, De Cat J, Desloovere K (1997). The use of botulinum toxin A in the treatment of gait disorders in CP: a multilevel approach [abstract]. Gait Posture 6:274. 